\title{
Blood Flow Regulating Structures in the Avian Kidney
}

\author{
H. Ditrich*
}

\author{
*Institute for Zoology, University of Vienna, Althanstrasse 14, A-1090 Vienna, Austria
}

The vascular supply of the kidney of birds is far more complicated than in mammals. In addition to arterial input and venous drainage, there is a prominent venous portal system, as in all nonmammalians (Fig. 1). The latter delivers blood from the leg (V. iliaca externa - Ua), the lateral pelvis $(\mathrm{V}$. ischiadica $-U b$ ) and the cloacal region (V. hypogastrica and V. pudenda - $U c$ and $U d$ ). This blood then passes the peritubular capillaries of the kidney, providing the substrate for uric acid excretion, the main avian nitrogenous waste product. However, not all of the portal blood passes through the renal capillaries. A variable proportion bypasses the kidney via the common iliac / posterior caval vein $(V C P)$ and the coccygo-mesenteric vein $(V C M)$.

Vascular corrosion casting is the method of choice for studies on delicate vascular systems. As previously shown [e.g., 1], not only the course of the vessels but also their relative diameters and certain structures of the vessel walls can be replicated. In this study, vascular corrosion casting and scanning electron microscopy (SEM) were applied to study some of the structures responsible for flow control in the avian kidney.

Adult animals of the domestic chicken (Gallus domesticus - four specimens), the mallard (Anas platyrhynchus - eleven specimens) and the turtle dove (Streptopelia roseogrisea - nine specimens) were used for this study. The animals were either injected with Mercox ${ }^{\circledR}$ (Jap. Vilene Co.) diluted with 20\% methyl-methacrylate monomer as described previously [2], or perfusion-fixed with $2 \%$ formaldehyde and $0.5 \%$ glutardialdehyde and then processed for conventional light- and transmission electron microscopy or critical point dried (CPD) for SEM.

The main shunt of portal blood from the kidney is regulated by the well studied valve at the external/ common iliac junction (Fig. $2-R 3$ ). (Partial) closure of this valve forces blood into the anterior $(R 2)$ and middle lobes $(R 1)$ of the kidney. Physiological studies show that this valve is under neuronal (noradrenalin/acetylcholine) control [3]. The role of the coccygo-mesenteric vein (VCM) is less clear. Tracer studies indicate that this vessel may be able to reverse it's flow [4, 5]. I. e., instead of forming a bypass for the blood from the dorso-posterior region that does not enter the renal portal system, this vessel could serve as an additional renal portal vein. In any case, a flow-regulating structure (R7) has to be postulated at the junction of the right (I7) and left posterior renal portal veins with the coccygo-mesenteric vein. Despite minor anatomical differences in the three investigated species, preliminary data show that distinct surface impressions (Fig. 3) can be found on corrosion casts at the emergence of the coccygo-mesenteric vein. The variable depth of these imprints indicates, that these structures are at the site of constricting muscles in the vascular wall.

[1] K.C. Hodde et al., Scanning Microsc. 4 (1990) 693.

[2] H. Ditrich and H. Splechtna, Scanning Microsc. 1 (1987) 1339.

[3] A.R. Akester, The cardiovascular system. In: Physiology and biochemistry of the domestic fowl. (Eds.: D.J. Bell, B.M. Freeman), Academic Press, New York, 1984.

[4] K. Shimada and P.D. Sturkie, Jap. J. Vet. Sci. 35 (1973) 57.

[5] B.W. Oelofsen, Zool. Africana 8 (1973) 41. 


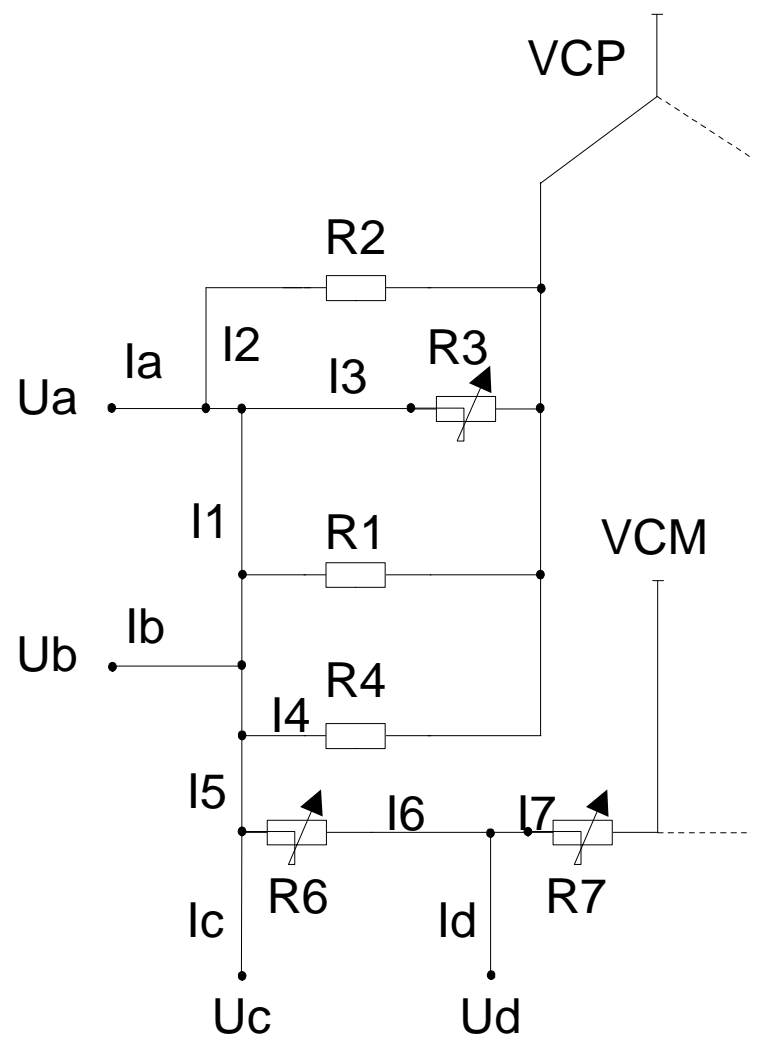

Fig. 1. Diagram of the venous system of the right kidney of birds. Note that the resistance at $R 6$ is based on theoretical considerations and has yet to be confirmed.

Fig. 3. Corrosion cast of the junction of the two posterior renal portal veins with the coccygomesenteric vein of the duck. Note the deep imprints in the resin surface, indicating circular muscles in the vessel wall (arrow).

(light microscope view);

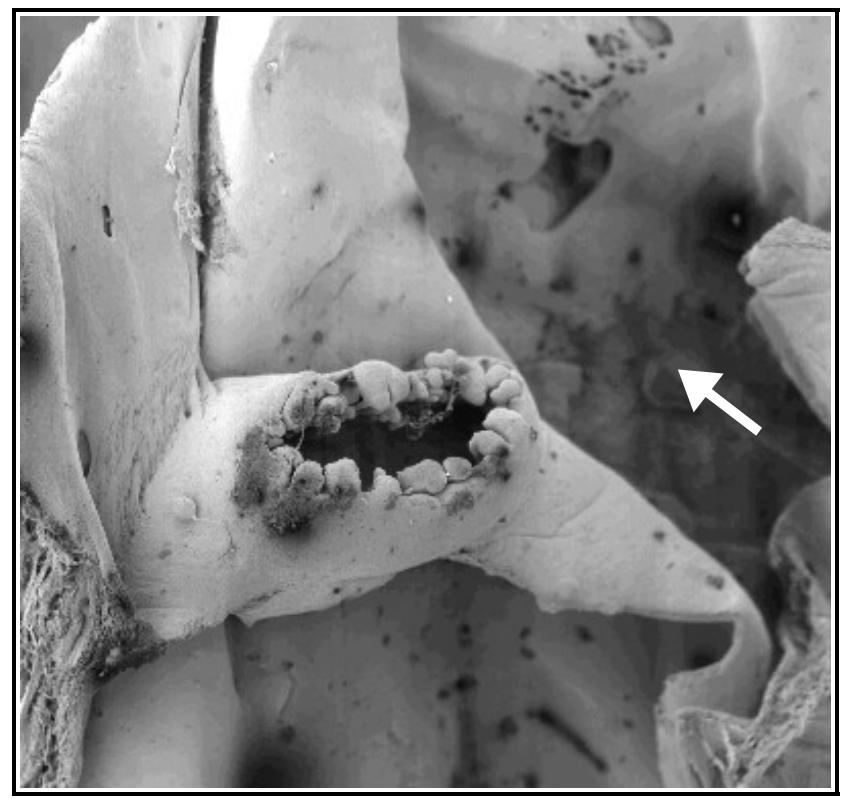

Fig. 2. Bypass valve $(R 3)$ at the external(bottom) / common iliac (top) junction in the dove. The efferent renal vein is joining from the right (arrow). (CPD tissue in SEM,);

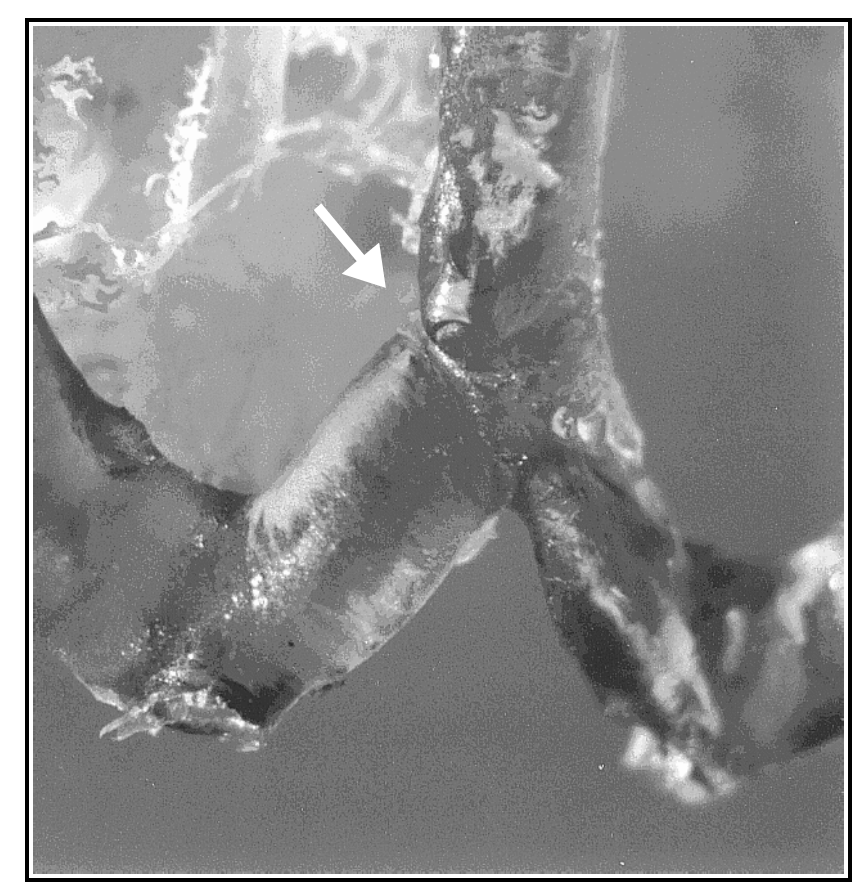

